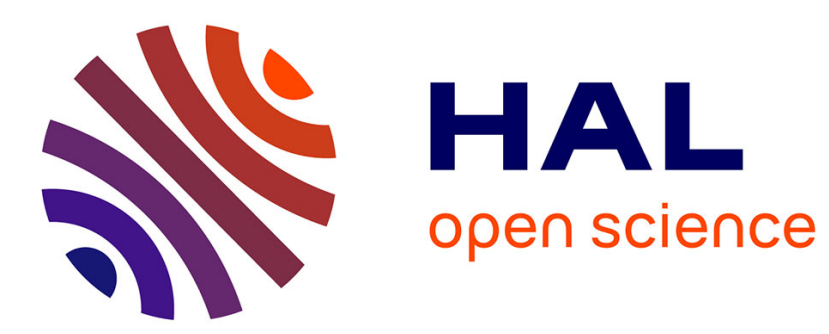

\title{
Market composition and price informativeness in a large market with endogenous order types \\ Edouard Challe, Edouard Chretien
}

\section{To cite this version:}

Edouard Challe, Edouard Chretien. Market composition and price informativeness in a large market with endogenous order types. 2014. hal-01060216

\section{HAL Id: hal-01060216 https://hal.science/hal-01060216}

Preprint submitted on 3 Sep 2014

HAL is a multi-disciplinary open access archive for the deposit and dissemination of scientific research documents, whether they are published or not. The documents may come from teaching and research institutions in France or abroad, or from public or private research centers.
L'archive ouverte pluridisciplinaire HAL, est destinée au dépôt et à la diffusion de documents scientifiques de niveau recherche, publiés ou non, émanant des établissements d'enseignement et de recherche français ou étrangers, des laboratoires publics ou privés. 
MARKET COMPOSITION AND PRICE INFORMATIVENESS IN A LARGE MARKET WITH ENDOGENOUS ORDER TYPES

\author{
Edouard CHALLE \\ Edouard CHRETIEN
}

Cahier n $^{\circ}$ 2014-19

\title{
DEPARTEMENT D'ECONOMIE \\ Route de Saclay \\ 91128 PALAISEAU CEDEX \\ (33) 169333033 \\ http://www.economie.polytechnique.edu/ \\ mailto:chantal.poujouly@polytechnique.edu
}




\title{
MARKET COMPOSITION AND PRICE INFORMATIVENESS IN A LARGE MARKET WITH ENDOGENOUS ORDER TYPES*
}

\author{
Edouard Challe ${ }^{\dagger} \quad$ Edouard Chrétien ${ }^{\ddagger}$
}

July 28,2014

\begin{abstract}
We analyse the joint determination of price informativeness and the composition of the market by order type in a large asset market with dispersed information. The market microstructure is one in which informed traders may place market orders or full demand schedules and where market makers set the price. Market-order traders trade less aggressively on their information and thus reduce the informativeness of the price; in a full market-order market, price informativeness is bounded, whatever the quality of traders' information about the asset's dividend. When traders can choose their order type and demand schedules are (even marginally) costlier than market orders, then market-order traders overwhelm the market when the precision of private signals goes to infinity. This is because demand schedules are substitutes: at high levels of precision, a residual fraction of demand-schedule traders is sufficient to take the trading price close to traders' signals, while the latter is itself well aligned with the dividend. Hence, the gain from trading conditional on the price (as demand-schedule traders do) in addition to one's own signal (as all informed traders do) vanishes.
\end{abstract}

\section{INTRODUCTION}

We analyse the joint determination of price informativeness and the composition of the market by order type in a large, competitive asset market with dispersed information. The market microstructure we consider is one in which informed traders may place either full demand schedules or more basic market orders, i.e., order to sell or buy a fixed quantity of assets unconditional on the execution price. ${ }^{1}$ There are also "noise" traders who prevent the asset price from being fully revealing whenever the precision of private signals is bounded, as in, e.g., Grossman and Stiglitz (1976, 1980), Diamond and Verrecchia (1981) and others. After informed and noise traders have placed their orders, the trading price is set by a competitive, utility-maximising market making sector. We characterise the trading intensities associated with each order type, the ex ante utilities that they generate for the concerned traders (hence their preference for a particular type of order), and ultimately how the composition of the market interacts with the informativeness of the price.

We first consider the case where exogenous measures of demand-schedule and market-order traders coexist in the market. In a pure market-order market (as in, e.g., Vives, 1995), the informativeness of the price is bounded above, however precise private information about the dividend is. In contrast, whenever demand-schedule traders are in positive mass the informativeness of the price is unbounded as the quality of private information goes to infinity. The reason for this difference lies in the way private information is incorporated into the price in either case. Because

\footnotetext{
${ }^{*}$ We are particularly grateful to two anonymous referees for their comments and to the editors for their guidance. Edouard Challe acknowledges the support of chaire FDIR. All remaining errors are ours.

${ }^{\dagger}$ CNRS (UMR 7176), Ecole Polytechnique, CREST and Banque de France; Email: edouard.challe@gmail.com.

${ }^{\ddagger}$ CREST; Email: edouard.chretien.2008@polytechnique.org.

${ }^{1}$ See Brown and Zhang (1997), Wald and Horrigan (2005) and Vives (2008) for further disscussion of the importance of market orders in actual asset markets.
} 
market-order traders face price risk - since their orders are unconditional on the effective trading price-, they trade less aggressively on their private information than demand-schedule traders, which reduces the informativeness of the price. In contrast, demand-schedule traders are insulated from price risk, so their trading intensity grows without bound as their private information becomes infinitely precise; in the limit, they perfectly align the trading price of the asset with the dividend (formally, the trading price is at least as informative of the dividend as the signals received by informed traders, and sometimes more).

Motivated by these observations, we examine informed traders' choices of order type and the impact of these choices on the composition of the market and the informativeness of the price. Since demand schedules are more complex than market orders (due to the full conditionality of the amount of trade on the price), we assume that they are more costly, at least marginally. Our main result is that, when the precision of private signals is large, then the equilibrium is necessarily interior (i.e., market-order and demand-schedule traders are both in strictly positive measures), but market-order traders overwhelm the market (i.e., their measure tends to one as precision goes to infinity). In other words, when the quality of information is high, the gain from conditioning one's trades on the price (as demand-schedule traders do) in addition to conditioning on one's own signal only (as market-order traders do) vanishes -and thus falls short of the cost, however small, for most traders.

There are two potential reasons for which this could be the case, and it is the purpose of the information structure that we assume -with potentially correlated noise in the signals received by informed traders- to disentangle them. First, knowledge of the price could become less and less valuable because one's own signal becomes more and more aligned with the information of others as the quality of private information improves (since all signals then get closer to the true value of the dividend). In other words, the advantage of acquiring information about the distribution of the signals received by others through the price (as in, e.g., Grossman and Stiglitz, 1976; Diamond and Verrecchia, 1981) is reduced when this distribution tightens, and vanishes in the limit. The other reason why knowing the price could become less valuable when private information become very precise is related to the price impact of noise trading. Demand-schedule traders trade against observed discrepancies between the trading price and their signals, and their trading intensity rises with the precision of the signal. As this precision goes to infinity, they trade so aggressively against noise traders that they effectively close the gap between the trading price and the dividend. By eliminating noise trader risk, demand-schedule traders reduce the value of knowing the price in addition to the signal itself. Considering the full spectrum of signal correlations allows us to identify which of the two informational roles of the price drives our main result. We show that the use of demand schedules vanishes at high levels of information precision even when signals are perfectly correlated, i.e. when information about the dividend is public. In this situation the price no longer plays any role as an aggregator of dispersed information. It follows that it is the reduction in the impact of noise trading on the equilibrium price that explains why knowledge of the price loses value as the information about the dividend becomes very accurate. Put differently, our analysis uncovers a form of substituability between demand schedules: when signals about the dividend are accurate, a small fraction of demand-schedule traders is enough to keep the price close to the signals they receive, which are themselves close to the true value of the dividend; hence, it is less 
useful to know the price in addition to one's own signal, so the incentive to purchase a demandschedule is reduced. ${ }^{2}$ In this sense, the pure demand schedule specification (the benchmark in the literature on price informativeness) is not innocuous and may not be stable to plausible changes in the microstructure of the market (here: the availability of a simpler, but cheaper, alternative order type).

Our analysis relates to at least two strands of the literature: one that explores the properties of asset prices under alternative order types and market microstructures, and one that studies the joint determination of information acquisition and equilibrium prices. The focus on market orders -as opposed to limit orders, stop orders or full demand schedules- in the market microstructure literature can be traced back to Vives (1995), Medrano (1996) and Brown and Zhang (1997). ${ }^{3}$ Vives (1995) studies a pure market-order market while Vives (2008) considers a market with exogenous sets of trader types. Medrano (1996) analyses the order choice of a single monopolistic trader, in the tradition of the "insider trading" literature. In contrast, we consider the endogenous determination of the sets of market-order versus demand-schedule traders in a large, competitive asset market. Brown and Zhang (1997) study traders' order choice in a large market, but in their model those who do not place market orders are "dealers" who observe the order flow but are uninformed about the asset dividend. The interest in the joint determination of equilibrium prices and information acquisition started with Grossman and Stiglitz (1980), followed by Verrecchia (1982) and more recently Barlevy and Veronesi (2000), Peress (2010) and Vives (2013). While this literature has traditionally focused on information acquisition about asset payoffs, our focus is on traders' willingness to purchase an information set that includes the trading price (as is the case with a demand schedule) -in addition to a free signal about the asset payoff.

Many of our results follow from the fact that market-order traders trade less aggressively than demand-schedule traders and thereby reduce price informativeness. Let us stress that this is by no means the only reason why price informativeness may be impeded relative to the competitive, full demand-schedule benchmark. First, there might be some unlearnable residual uncertainty about the dividend, a possibility explored by Angeletos and Werning (2006, pp. 1734-5) in a somewhat different context. They show that this causes the precision of the price signal to be bounded above, whatever the precision of the private signals on the learnable part of the dividend. Market frictions may also limit traders' reaction to their information and thus price informativeness. For example, short-sale constraints limit traders' responsiveness to bad news (Miller, 1977; Diamond and Verrecchia, 1987; Bai et al. 2006). Similarly, under imperfect competition traders reduce their trading intensity so as to avoid revealing their private information (Kyle, 1989). Our approach is closer in spirit to the latter contribution in that limited trading aggressiveness follows from the microstructure of the market, rather than outside restrictions about the learnability of the information or the size of trades.

Section 2 presents the trading game. Section 3 analyses the case where order types are exoge-

\footnotetext{
${ }^{2}$ This relies on demand schedule traders not reaching measure zero, in which case the price would no longer be well aligned with their signals. We show, however, that this cannot be the case under endogenous order types. The reason is that in a full market-order market the aggressiveness of informed traders is bounded above, hence these traders no longer eliminate noise trader risk when signals are very precise; this makes demand schedules valuable again and ensures that demand-schedule traders have strictly positive measure.

${ }^{3}$ See Medrano (1996) and the references therein for a detailed disucssion of the early literature on market orders, and Vives (2008) for the more recent papers.
} 
nous, and Section 4 that where they are endogenous. Section 5 concludes the paper.

\section{THE MODEL}

We consider the following competitive model of asset trading. There are two assets: (i) a riskless bond in perfectly elastic supply and paying out a constant interest rate; and (ii) a risky asset with trading price $p$ and terminal dividend $\theta$, where $\theta$ is drawn from the distribution $\mathcal{N}\left(\bar{\theta}, \alpha_{\theta}^{-1}\right), \alpha_{\theta}>0$, before any trading takes place. Traders know the distribution of $\theta$ but not its realisation.

There is a continuum of informed traders $i \in I=[0,1]$, each of whom gets a free private, noisy signal about the dividend $x_{i}=\theta+\alpha_{x}^{-1 / 2} \xi_{i}$, with $\alpha_{x}>0, \xi_{i} \sim \mathcal{N}(0,1)$ and $\operatorname{cov}\left(\xi_{i}, \theta\right)=0$. We allow the noise in the private signals to be cross-correlated and parameterise this property by the correlation coefficient $\rho \in[0,1]$. We adopt the convention that the average of i.i.d. random variables with mean zero is zero and we let $\tilde{\xi} \equiv \int_{0}^{1} \xi_{i} \mathrm{~d} i \sim \mathcal{N}(0, \rho)$ and $\eta_{i} \equiv \xi_{i}-\tilde{\xi} \sim \mathcal{N}(0,1-\rho)$ denote, respectively, the aggregate and idiosyncratic components of the noise in the private signals. ${ }^{4}$ As $\rho$ gets closer to one the information received by informed traders gets increasingly shared between themselves; when $\rho=1$ private signals are perfectly correlated and the private signal $\xi_{i}$ is just the public signal $\tilde{\xi}$. In contrast, as $\rho$ goes down then the noise components become increasingly uncorrelated across traders, and we recover the usual specification with uncorrelated informational noise with $\rho=0$. We may rewrite the private signal $x_{i}$ as follows:

$$
x_{i}=\theta+\alpha_{x}^{-1 / 2} \tilde{\xi}+\alpha_{x}^{-1 / 2} \eta_{i},
$$

which implies that $x_{i}$ provides a noisy signal about $\theta+\alpha_{x}^{-1 / 2} \tilde{\xi}$ (with neither $\theta$ nor $\tilde{\xi}$ being individually observed).

Aside from informed traders, there are noise traders in the market, which place a net asset demand for the risky asset $\varepsilon \sim \mathcal{N}\left(0, \alpha_{\varepsilon}^{-1}\right)$, with $\alpha_{\varepsilon}>0$. Following Vives $(1995,2008)$, we consider a competitive market microstructure wherein (a) all or some traders place market orders (rather than full demand schedules), and (b) a (competitive, risk-neutral) market-making sector sets the price $p$. In contrast to a demand schedule, a market order is conditional on the private signal $x_{i}$ but not on the execution price $p$; once placed, it is executed irrevocably at whatever value of $p$ is set by market makers. The market-making sector observes the order book $L(\cdot)$ emanating from informed and noise traders and sets the price $p$; competition among risk-neutral market makers then causes them to undercut each other until $p=\mathbb{E}[\theta \mid L(\cdot)]$. Note that $L(\cdot)$ is itself a function of $p$ whenever a positive mass of informed traders places demand schedules.

We introduce a general correlation structure for the signals received by informed traders for the following reason. In our model placing a full demand schedule (as opposed to a market order) allows a trader to trade conditionally on the price, which is valuable for two very distinct reasons. First, the price aggregates dispersed private information and thus provides additional information about the fundamental relative to one's own signal. Second, it provides information about the realised amount of noise trading; that is, it effectively allows a trader to partly hedge noise trader risk (to which a market-order trader is exposed). For $\rho \in[0,1)$, these two informational roles of price are present, but when $\rho=1$ only the second one is. Hence, considering the full correlation

\footnotetext{
${ }^{4}$ See Vives (2013) for a model with a similar form of cross-correlation between agent types (formulated in terms of the marginal utility of a good, rather than a direct signal about an asset payoff).
} 
spectrum $[0,1]$ will shed light on which role of the price really matters for our results.

Let $M \subset I$ be the set of market-order traders and $I \backslash M$ the complementary set of demandschedules traders, of measure $\nu \equiv \int_{I \backslash M} \mathrm{~d} i \in[0,1]$. We will consider both the case where $M$ and $I \backslash M$ are exogenous (Section 3) and that where they are endogenous (Section 4). All informed traders have zero initial wealth (this is without loss of generality) and preferences $V\left(w_{i} ; \gamma_{i}\right)=-e^{-\gamma_{i} w_{i}}$, where $\gamma_{i}>0$ and $w_{i}=(\theta-p) k_{i}$ are the risk aversion coefficient and terminal wealth of trader $i$, respectively. We denote by $\gamma_{I}^{-1} \equiv \int_{I} \gamma_{i}^{-1} \mathrm{~d} i$ the average risk tolerance of informed traders. We rank informed traders in nondecreasing order of risk aversion and define the nondecreasing function $\gamma$ : $[0,1] \rightarrow \mathbb{R}_{+}$. Finally, we assume that (i) $\gamma_{i}$ is increasing and continuous in $i$ and such that $\gamma_{0}>0$; and (ii) $\gamma_{i}^{-1}$ is independent of $\xi_{i}-\tilde{\xi}$, i.e., $\forall J \subset I, \int_{J} \gamma_{i}^{-1}\left(\xi_{i}-\tilde{\xi}\right) \mathrm{d} i=0$ a.s.

Definition. A Bayesian equilibrium of the trading game is a pair of investment functions for demand-schedule $\left(k_{I \backslash M}\left(x_{i}, p ; \gamma_{i}\right)\right)$ and market-order $\left(k_{M}\left(x_{i} ; \gamma_{i}\right)\right)$ traders as well as a price function $p(\theta, \tilde{\xi}, \varepsilon)$ such that (i) $k_{I \backslash M}(\cdot)$ and $k_{M}(\cdot)$ maximise informed traders' expected utility:

$$
\begin{gathered}
\forall i \in I \backslash M, k_{I \backslash M}\left(x_{i}, p ; \gamma_{i}\right) \in \arg \max _{k \in \mathbb{R}} \mathbb{E}\left[V\left((\theta-p) k ; \gamma_{i}\right) \mid x_{i}, p\right], \\
\forall i \in M, k_{M}\left(x_{i} ; \gamma_{i}\right) \in \arg \max _{k \in \mathbb{R}} \mathbb{E}\left[V\left((\theta-p) k ; \gamma_{i}\right) \mid x_{i}\right]
\end{gathered}
$$

and (ii) the market-making sector sets the price $p=\mathbb{E}[\theta \mid L(\cdot)]$, where

$$
L(p)=\int_{I \backslash M} k_{I \backslash M}\left(x_{i}, p ; \gamma_{i}\right) \mathrm{d} i+\int_{M} k_{M}\left(x_{i} ; \gamma_{i}\right) \mathrm{d} i+\varepsilon .
$$

We then have the following lemma:

Lemma 1. The trading game has a unique linear Bayesian equilibrium, which is characterised by:

- The investment functions

$$
k_{I \backslash M}\left(x_{i}, p ; \gamma_{i}\right)=\frac{\alpha_{\theta} \rho \bar{\theta}+\alpha_{x} x_{i}-\left(\alpha_{x}+\alpha_{\theta} \rho\right) p}{\gamma_{i}\left(1+(1-\rho) \rho B^{2} \alpha_{\varepsilon} / \alpha_{x}\right)} \text { and } k_{M}\left(x_{i} ; \gamma_{i}\right)=\frac{\beta\left(x_{i}-\bar{\theta}\right)}{\gamma_{i}},
$$

with

$$
\beta=\left[\left(\alpha_{x}^{-1}+\alpha_{\theta}^{-1}\right)\left(1+B^{2} \rho \alpha_{x}^{-1} \alpha_{\varepsilon}\right)-\left(B^{2} \alpha_{\varepsilon}\left(1+\rho \alpha_{x}^{-1} \alpha_{\theta}\right)+\alpha_{\theta}\right)^{-1}\right]^{-1}
$$

- The price function

$$
p(\theta, \tilde{\xi}, \varepsilon)=(1-\lambda B) \bar{\theta}+\lambda B\left(\theta+\alpha_{x}^{-1 / 2} \tilde{\xi}+B^{-1} \varepsilon\right),
$$

with

$$
\lambda=B \alpha_{\varepsilon}\left[B^{2} \alpha_{\varepsilon}\left(1+\rho \alpha_{\theta} / \alpha_{x}\right)+\alpha_{\theta}\right]^{-1} .
$$

In those functions, $B>0$ is the unique real solution to the cubic equation:

$$
B=\frac{\alpha_{x} \nu \gamma_{I \backslash M}^{-1}}{1+(1-\rho) \rho B^{2} \alpha_{\varepsilon} / \alpha_{x}}+\frac{(1-\nu) \gamma_{M}^{-1}}{\left(\alpha_{x}^{-1}+\alpha_{\theta}^{-1}\right)\left(1+B^{2} \rho \alpha_{\varepsilon} / \alpha_{x}\right)-\left[B^{2} \alpha_{\varepsilon}\left(1+\rho \alpha_{\theta} / \alpha_{x}\right)+\alpha_{\theta}\right]^{-1}}
$$


where $\gamma_{I \backslash M}^{-1} \equiv \nu^{-1} \int_{I \backslash M} \gamma_{i}^{-1} \mathrm{~d} i$ and $\gamma_{M}^{-1} \equiv(1-\nu)^{-1} \int_{M} \gamma_{i}^{-1} \mathrm{~d} i$ are the average risk tolerance coefficients of demand-schedule and market-order traders, respectively.

Lemma 1 generalises the trading game in Vives (2008, Sec. 4.3) in two directions: i) heterogenous risk aversion, and ii) correlated noise in the private signals. Heterogeneity in risk aversion is the dimension along which informed traders sort themselves into demand-schedule versus marketorder traders in Section 4. The possibility that private informational noise be correlated will imply that our results do not depend on whether the signal $x_{i}$ is private $(\rho<1)$ or public $(\rho=1)$.

Equation (7) implies that observing $p$ is equivalent to observing $\theta+\alpha_{x}^{-1 / 2} \tilde{\xi}+B^{-1} \varepsilon$. Hence $p$ provides a public signal that is jointly informative of $\theta, \tilde{\xi}$ and $\varepsilon$. Note that the signal extraction problem faced by demand-schedule traders is more involved when $\rho \in(0,1)$ than when $\rho \in\{0,1\}$. When $\rho=0$ we have $\tilde{\xi}=0$ a.s., hence $p$ provides a signal about $\theta$ with noise $B^{-1} \varepsilon$. When $\rho=1$, the public signal $\theta+\alpha_{x}^{-1 / 2} \tilde{\xi}$ is observed (i.e., $\eta_{i}=0 \forall i$ ) jointly with $p$, hence $B^{-1} \varepsilon$ can be perfectly inferred (see (7) again). In contrast, when $\rho \in(0,1)$ then $\tilde{\xi}$ must be inferred together with $\theta$ from the observation of $p$. This joint signal extraction problem manifests itself by a greater residual uncertainty about $\theta$ (conditional on a given signal $x_{i}$ ) when $\rho \in(0,1)$ than when $\rho \in\{0,1\}$, which lowers the responsiveness of the demand for assets by demand-schedule traders to their signal. ${ }^{5}$

\section{EXOGENOUS TRADER TYPES}

We first analyse price informativeness at high signal precision when the distribution of informed traders across types is exogenous. We then have the following proposition:

Proposition 1. (a) In a pure market-order market $(\nu=0)$, the informativeness of the price signal is bounded above; formally, $\alpha_{p} \underset{\alpha_{x} \rightarrow \infty}{\rightarrow} B_{0}^{2} \alpha_{\varepsilon}<+\infty$, where $B_{0}>0$ uniquely solves $\gamma_{M} B_{0}\left(\alpha_{\theta}^{-1}-\right.$ $\left.\left(\alpha_{\theta}+\alpha_{\varepsilon} B_{0}^{2}\right)^{-1}\right)=1$. (b) Whenever there is a positive mass of demand-schedule traders $(\nu>0)$, then the precision of the price signal is unbounded as $\alpha_{x} \rightarrow \infty$; more specifically,

$$
\alpha_{p} \underset{\alpha_{x} \rightarrow+\infty}{\sim} \mathbf{1}_{\rho>0}\left(\alpha_{x} / \rho\right)+\mathbf{1}_{\rho=0}\left(\nu \alpha_{x} / \gamma_{I \backslash M}\right)^{2} \alpha_{\varepsilon}
$$

Proposition 1 shows that the speed of information aggregation as $\alpha_{x} \rightarrow+\infty$ depends on both the cross-correlation of informed traders' signals and the share of market-order traders among them. First, whenever $\nu>0$, then information aggregation is less effective when $\rho>0$ than when $\rho=0$ (the informativeness of the price $\alpha_{p}$ grows at the rate of $\alpha_{x}$ in the former case but at the rate of $\alpha_{x}^{2}$ in the latter). Second, information aggregation is less effective when $\nu=0$ than otherwise (the informativeness of $p$ is bounded above as $\alpha_{x} \rightarrow \infty$ in the former case, not in the latter).

The intuition for the second result follows from our assumed information structure and its implications for the Bayesian updating problem of demand-schedule traders. As stressed above, this problem is more involved when $\rho>0$ than when $\rho=0$ : in the latter case the quality of the price signal $p$ is only blurred by the extent of noise trading $\varepsilon$, while in the former it is also blurred by the common informational noise component $\tilde{\xi}$. As $\alpha_{x}$ increases and private signals become more and more aligned with $\theta$, the impact of $\varepsilon$ on $p$ diminishes (since $\alpha_{\varepsilon}$ is constant) but that of $\tilde{\xi}$ does

\footnotetext{
${ }^{5}$ This effect shows up in the fact that the multiplier $\alpha_{x} /\left[\gamma_{i}\left(1+(1-\rho) \rho B^{2} \alpha_{\varepsilon} / \alpha_{x}\right)\right]$ in the investment function of demand-schedule traders (see (5)) has the term $\rho(1-\rho)$ in the denominator. This product is equal to zero for $\rho \in\{0,1\}$ but is positive for $\rho \in(0,1)$ and is maximal at $\rho=1 / 2$.
} 
not (since its precision $1 / \mathbb{V}\left(\alpha_{x}^{-1 / 2} \tilde{\xi}\right)=\alpha_{x} / \rho$ increases at the rate of $\left.\alpha_{x}\right)$. In the special case where $\rho=0$ the informativeness of $p$ grows very rapidly (at the rate of $\alpha_{x}^{2}$ ) because the common noise component effect is absent; whenever it is present, the quality of the price signal cannot grow at a rate faster than $\alpha_{x}$. This suggests that the usual specification where $\rho=0$ is somewhat special and that the conclusions drawn from it are not necessarily robust. Here it implies that as $\alpha_{x}$ grows large then $x_{i}$ (whose precision grows at rate $\alpha_{x}$ ) looses value relative to $p$ (whose precision grows at the rate of $\alpha_{x}^{2}$ ); eventually, demand-schedule traders only base their Bayesian estimate of $\theta$ on $p$. In contrast, when $\rho>0$ and the informativeness of $p$ grows at the same rate as $\alpha_{x}$, then $x_{i}$ and $p$ keep constant weights in the computation of the posterior mean of $\theta$ as $\alpha_{x} \rightarrow+\infty$.

The intuition for the first result in the proposition (i.e., that price informativeness is bounded when $\nu=0)$ is as follows. In a pure demand-schedule market $(\nu=1)$, informed traders can condition their trades on $p$, so the only source of risk they face concerns the true value of $\theta$. As the precision of private signals increases, informed traders collectively trade more and more aggressively against any discrepancy between $p$ and $\theta$. Formally, from (1) and Lemma 1 the total asset demand by informed traders in a pure demand-schedule market can be written as:

$$
\int_{I}\left(\frac{\alpha_{\theta} \rho \bar{\theta}+\alpha_{x}\left(\theta+\alpha_{x}^{-1 / 2} \tilde{\xi}+\alpha_{x}^{-1 / 2} \eta_{i}\right)-\left(\alpha_{x}+\alpha_{\theta} \rho\right) p}{\gamma_{i}\left(1+(1-\rho) \rho B^{2} \alpha_{\varepsilon} / \alpha_{x}\right)}\right) \mathrm{d} i=B\left(\theta-p+\frac{\alpha_{\theta} \rho}{\alpha_{x}}(\bar{\theta}-p)+\alpha_{x}^{-1 / 2} \tilde{\xi}\right),
$$

where $B$ uniquely solves $B \gamma_{I}\left(1+(1-\rho) \rho B^{2} \alpha_{\varepsilon} / \alpha_{x}\right)=\alpha_{x}$ (since $I \backslash M=I$ and hence $\nu=1$ ). The latter expression implies that $B \rightarrow+\infty$ as $\alpha_{x} \rightarrow+\infty$, and thus, by equations (7)-(8), that $p \rightarrow \theta$ as $\alpha_{x} \rightarrow+\infty$-i.e., in the limit $p$ becomes perfectly informative of $\theta$. In contrast, in a pure market-order market $(\nu=0)$ informed traders do not condition their trades on $p$ and hence face a residual payoff risk even as the $x_{i}$ s get more and more informative of $\theta$. This risk leads market-order traders to trade relatively less aggressively on the basis of their private signal, which limits the amount of information that is transmitted into the price. Formally, from Lemma 1 again the total asset demand by informed traders in a pure market-order market is:

$$
\int_{I}\left(\frac{\beta\left(\theta+\alpha_{x}^{-1 / 2} \tilde{\xi}+\alpha_{x}^{-1 / 2} \eta_{i}-\bar{\theta}\right)}{\gamma_{i}}\right) \mathrm{d} i=\frac{\beta\left(\theta+\alpha_{x}^{-1 / 2} \tilde{\xi}-\bar{\theta}\right)}{\gamma_{M}}
$$

where $\beta$ is given by (6) $B$ solves (since $\nu=0$ ):

$$
\left(\alpha_{x}^{-1}+\alpha_{\theta}^{-1}\right)\left(1+B^{2} \rho \alpha_{\varepsilon} / \alpha_{x}\right)-\left(B^{2} \alpha_{\varepsilon}\left(1+\rho \alpha_{\theta} / \alpha_{x}\right)+\alpha_{\theta}\right)^{-1}=1 /\left(\gamma_{M} B\right)
$$

In this situation, as $\alpha_{x} \rightarrow+\infty$ we have $B \rightarrow B_{0}(>0)$, where $B_{0}$ is the unique solution to $\left(\alpha_{\theta}^{-1}-1 /\left(B^{2} \alpha_{\varepsilon}+\alpha_{\theta}\right)\right) \gamma_{M} B=1$. By implication, the trading intensity of market-order traders is bounded above as $\alpha_{x} \rightarrow+\infty$. From (7), as $\nu=0$ and $\alpha_{x} \rightarrow+\infty$ we have:

$$
p \rightarrow \frac{\alpha_{\theta} \bar{\theta} / \alpha_{\varepsilon}+B_{0}^{2}\left(\theta+B_{0}^{-1} \varepsilon\right)}{B_{0}^{2}+\alpha_{\theta} / \alpha_{\varepsilon}} .
$$

Thus, asymptotically observing the price is equivalent to observing $\theta+B_{0}^{-1} \varepsilon$, i.e., $p$ provides a signal about $\theta$ with precision $B_{0}^{2} \alpha_{\varepsilon}<+\infty$. The intermediate case $\nu \in(0,1)$ retains the main properties of the pure demand-schedule case, because any positive measure of demand-schedule 
traders is sufficient for their trading aggressiveness (which is unbounded as $\alpha_{x} \rightarrow+\infty$ ) to eliminate the impact of $\varepsilon$ on $p$. As we show next, this intermediate case is that which necessarily arises in equilibrium when traders are free to choose their order type and the quality of information is high.

\section{Endogenous trader types}

We now analyse traders' choice of order type and determine the equilibrium sets $M$ and $I \backslash M$. The basic tradeoff is that a demand schedule isolates a trader from price risk, but requires the trader to place a large (infinite) number of limit orders to generate complete conditionality of trades on the execution price. We capture this tradeoff by normalising the cost of a market order to zero and setting that of a full demand schedule to $c>0$. We know from the CARA-Normal model that the value function associated with the information set $G_{i}$ is:

$$
W\left(G_{i} ; \gamma_{i}\right) \equiv \max _{k} \mathbb{E}\left[V\left(w_{i}-\kappa c\right) \mid G_{i} ; \gamma_{i}\right]=-\exp \left(-\frac{\mathbb{E}\left[\theta-p \mid G_{i}\right]^{2}}{2 \mathbb{V}\left[\theta-p \mid G_{i}\right]}+\kappa c \gamma_{i}\right)
$$

where $\kappa=1$ if $G_{i}=\left(x_{i}, p\right)$ (i.e., the trader places a full demand schedule) or $\kappa=0$ if $G_{i}=x_{i}$ (i.e., the trader places a market order). Let $W_{I \backslash M}\left(x_{i}, p ; \gamma_{i}\right)$ and $W_{M}\left(x_{i} ; \gamma_{i}\right)$ denote the expected utilities of a demand-schedule and a market order trader, respectively, with preferences $\gamma_{i}$ and conditional on their full information set (i.e., $x_{i}$ or $\left(x_{i}, p\right)$ ). There are two possible timing assumptions here, depending on whether we allow informed traders to choose their order type after ("timing 1") or before ("timing 2") observing $x_{i}$. Under timing 1 traders compare expected utilities conditional on $x_{i}$ (i.e., $W_{M}\left(x_{i} ; \gamma_{i}\right)$ and $\left.\mathbb{E}\left[W_{I \backslash M}\left(x_{i}, p ; \gamma_{i}\right) \mid x_{i}\right]\right)$, while under timing 2 they compare the same expected utilities integrated over $x_{i}$ (i.e., $\mathbb{E}\left[W_{M}\left(x_{i} ; \gamma_{i}\right)\right]$ and $\left.\mathbb{E}\left[W_{I \backslash M}\left(x_{i}, p ; \gamma_{i}\right)\right]\right){ }^{6}$ The following Lemma shows that the expected utility ratios are the same under the two timing assumptions, hence both lead to the same discrete choice of order type.

Lemma 2. The ratios of conditional and unconditional expected utilities are given by:

$$
\underbrace{\frac{W_{M}\left(x_{i} ; \gamma_{i}\right)}{\mathbb{E}\left[W_{I \backslash M}\left(x_{i}, p ; \gamma_{i}\right) \mid x_{i}\right]}}_{\text {timing 1 }}=\underbrace{\frac{\mathbb{E}\left[W_{M}\left(x_{i} ; \gamma_{i}\right)\right]}{\mathbb{E}\left[W_{I \backslash M}\left(x_{i}, p ; \gamma_{i}\right)\right]}}_{\text {timing 2 }}=\underbrace{\mathrm{e}^{-c \gamma_{i}} \sqrt{\frac{\mathbb{V}\left[\theta-p \mid x_{i}\right]}{\mathbb{V}\left[\theta-p \mid x_{i}, p\right]}}}_{\text {common value of the ratio }},
$$

where $\mathbb{V}\left[\theta-p \mid x_{i}, p\right]$ and $\mathbb{V}\left[\theta-p \mid x_{i}\right]$ are given by equations (A2) and (A4) in Appendix A.

In other words, when we move from timing 1 to timing 2 , both expected utilities change but in the same proportion, leaving the basic tradeoff between order types unchanged. It follows that under either timing informed trader $i$ places a demand schedule if and only if the relevant ratio is below or equal to one. Given the value of $\mathbb{V}\left[\theta-p \mid x_{i}\right] / \mathbb{V}\left[\theta-p \mid x_{i}, p\right]$, computed from equations (A2)

\footnotetext{
${ }^{6}$ The equilibria that we focus on under timing 1 are linear Bayesian equilibria with linear price functionals. In these equilibria informed traders choose their type on the basis of their risk aversion only (and not, say, on the level of their signal). Consequently, (i) the distribution of signals remains independent of that of risk aversion within each set $M$ and $I \backslash M$, even though these are determined after the signals are observed; and hence (ii) the equilibrium measure $\nu$ is uninformative of the dividend. Note that even in this timing $W_{I \backslash M}\left(x_{i}, p ; \gamma_{i}\right)$ is not known because it is a function of $p$, a random variable at the time the order type is chosen. In contrast $W_{M}\left(x_{i} ; \gamma_{i}\right)$ is known, since it is not conditional on the yet unknown value of $p$ (by the mere definition of a market order). This is why traders must compute $\mathbb{E}\left[W_{I \backslash M}\left(x_{i}, p ; \gamma_{i}\right) \mid x_{i}\right]$ and compare it with $W_{M}\left(x_{i} ; \gamma_{i}\right)$.
} 
and (A4) in the appendix, this is equivalent to:

$$
\begin{aligned}
\gamma_{i} & \leq \bar{\gamma}=\frac{1}{2 c}\left[\ln \left(\frac{1}{\alpha_{x}+\alpha_{\theta}}\left(\rho+\frac{\rho \alpha_{\theta}}{\alpha_{x}}+\frac{\alpha_{\theta}}{B^{2} \alpha_{\varepsilon}}\right)^{2}+\frac{\rho(1-\rho)}{\alpha_{x}}+\frac{1}{B^{2} \alpha_{\varepsilon}}\right)\right. \\
& \left.-\ln \left(\frac{1}{\alpha_{\theta}}\left(1-\left(1+\frac{\rho \alpha_{\theta}}{\alpha_{x}}+\frac{(1-\rho) \alpha_{\theta}}{\alpha_{x}+(1-\rho) B^{2} \alpha_{\varepsilon}}\right)^{-1}\right)\left(1+\frac{\rho \alpha_{\theta}}{\alpha_{x}}+\frac{\alpha_{\theta}}{B^{2} \alpha_{\varepsilon}}\right)^{2}\right)\right],
\end{aligned}
$$

where, from Lemma 1,

$$
\begin{aligned}
B & =\left(\frac{\alpha_{x}}{\left(1+(1-\rho) \rho B^{2} \alpha_{\varepsilon} / \alpha_{x}\right)}\right) \int_{0}^{\gamma^{-1}(\bar{\gamma})} \gamma_{i}^{-1} \mathrm{~d} i \\
& +\left(\left(\frac{1}{\alpha_{x}}+\frac{1}{\alpha_{\theta}}\right)\left(1+\frac{B^{2} \rho \alpha_{\varepsilon}}{\alpha_{x}}\right)-\frac{1}{B^{2} \alpha_{\varepsilon}\left(1+\rho \alpha_{\theta} / \alpha_{x}\right)+\alpha_{\theta}}\right)^{-1} \int_{\gamma^{-1}(\bar{\gamma})}^{1} \gamma_{i}^{-1} \mathrm{~d} i .
\end{aligned}
$$

with $\gamma^{-1}(\bar{\gamma})=0$ if $\bar{\gamma}<\gamma(0)$ and $\gamma^{-1}(\bar{\gamma})=1$ if $\bar{\gamma}>\gamma(1)$. For $\left(\alpha_{x}, \alpha_{\theta}, \alpha_{\varepsilon}, \rho\right) \in \mathbb{R}^{+3} \times[0,1]$ given, the properties of the $\gamma$ function imply that the solution $(\bar{\gamma}, B)$ to $(10)-(11)$, if it exists, can be of three types: it is either such that $\bar{\gamma} \in[\gamma(0), \gamma(1)]$, in which case the solution is interior (i.e., $M \neq \emptyset$ and $I \backslash M \neq \emptyset$ ); or $\bar{\gamma}<\gamma(0)$, so that the solution is corner and all traders place market orders (i.e., $(M, I \backslash M)=(I, \emptyset))$; or $\bar{\gamma}>\gamma(1)$ and all traders place demand schedules (i.e., $(M, I \backslash M)=(\emptyset, I))$. The intuition for the sorting of traders along the degree of risk aversion is that greater risk aversion lowers trading aggressiveness, hence the expected benefit from expanding the information set from $x_{i}$ to $\left(x_{i}, p\right)$. Proposition 2 states our main results under endogenous order types: ${ }^{7}$

Proposition 2. For any $\left(\alpha_{\theta}, \alpha_{\varepsilon}, \rho\right) \in \mathbb{R}^{+2} \times[0,1]$, and as $\alpha_{x} \rightarrow+\infty$, (a) the solution $(\bar{\gamma}, B)$ to (10)-(11) is unique; (b) both $M$ and $I \backslash M$ have strictly positive measure (i.e., the equilibrium is interior); (c) $\bar{\gamma} \rightarrow \gamma_{0}$ (i.e., market-order traders eventually overwhelm the market); (d) $\alpha_{p}$ goes to infinity as the same rate as $\alpha_{x}$; formally, defining the bijection $h_{\rho}: \mathbb{R}^{+} \rightarrow(0,1), h_{\rho}(x)=$ $\left(\rho+\frac{(1-\rho)}{1+(1-\rho) x}\right)\left(\rho+x^{-1}\right)^{-1}$ and $h_{\rho}^{-1}$ its inverse, we have

$$
\alpha_{p} \underset{\alpha_{x} \rightarrow \infty}{\sim} \frac{\alpha_{x}}{\rho+\left[h_{\rho}^{-1}\left(\mathrm{e}^{-2 \gamma_{0} c}\right)\right]^{-1}} .
$$

Our information structure gives us some intuition about why demand-schedule traders vanish as $\alpha_{x} \rightarrow+\infty$ (point (c)). In our analysis $p$ plays two distinct informational roles: it provides information about the distribution of signals received by the other informed traders, and about the net asset demand of noise traders. In the special case where information about $\theta$ is entirely public (i.e., $\rho=1$ ), there is nothing to learn from the other informed traders by observing $p$. However, trading conditional on $p$ is still valuable because this provides insurance against noise trader risk-to which market-order traders are exposed. That the crowding out of demand-schedule traders by market-order traders as $\alpha_{x} \rightarrow+\infty$ also takes place when $\rho=1$ suggests that when the quality of information is high the primary value of a demand schedule relative to a market order comes from its hedging role against noise trader risk, rather than its role at extracting dispersed information.

\footnotetext{
${ }^{7}$ Note from (10) that heterogeneity in $c$ is formally equivalent to heterogeneity in $\gamma$. To encompass both cases, rank traders in nondecreasing orders of $g(i) \equiv c(i) \gamma(i)$, assume that $g(i)$ is continuous, strictly increasing, that its reciprocal is continuous, and that $0<g(0)<g(1)<+\infty$; then solve for the marginal trader exactly in the same way as in the case where $c$ is homogenous.
} 
It follows that for the share of demand-schedule traders to vanish when signals become increasingly precise, it must be that the gain from hedging noise trader risk itself vanishes. But the reason for this is immediate: as the precision of information increases, demand-schedule traders trade more and more aggressively on their information. In so doing, they take $p$ closer and closer to their own signal $x_{i}$, which is itself closer and closer to $\theta$. Eventually, they completely eliminate noise trader risk, and thereby the relative benefit of a demand schedule.

This feature also explains why the equilibrium must necessarily be interior, i.e., why $I \backslash M$, whilst asymptotically vanishing, must always keep positive measure (point (b)). If it were not the case, then the market would be a full market-order market similar to that examined in Section 3. In this situation, the trading intensity of informed traders would be bounded above, hence the uncertainty about the dividend would be bounded below (see Proposition 1). But then noise trader risk would no longer be eliminated even at high levels of precision of the signals, and thus knowing the price (in addition to the signal about the dividend) to insure against noise trader risk would become valuable again. Demand schedules thus display a form of substituability: when information about $\theta$ is precise, then a positive but small measure of demand-schedule traders deters all the other traders from placing a demand schedule (however small $c$ is).

Finally, the informativeness of $p$ (point (d)) is closely related to the composition of the market (point (c)). As discussed in Section 3, market-order traders tend to reduce information aggregation. Consequently, the gradual crowding out of demand-schedule traders as $\alpha_{x} \rightarrow+\infty$ tends to reduce the pace of information aggregation, relative to the case with constant, exogenous shares of each type. For example, in the case where $\rho=0$ the precision of the price signal grows at the rate of $\alpha_{x}$, instead of $\alpha_{x}^{2}$ when the sets $M$ and $I \backslash M$ are exogenous.

\section{Concluding Remarks}

This paper has analysed the joint determination of price informativeness and the composition of the market in a large market with dispersed information. By allowing market-order and demandschedule traders to coexist, and by letting traders choose their preferred order type, the microstructure considered here is richer and more realistic than the pure demand schedule/Walrasian auctioneer specification. Our main result that the set of demand-schedule traders vanishes when signals become highly informative follows logically from the structure of the trading game, so we expect it to hold under more general assumptions than those we have assumed. For example, we have adopted the usual CARA-Normal framework, which is the only tractable specification under our information structure. However, nothing in our results seem to depend on a particular feature of preferences, at least in an obvious way; we thus conjecture that they would remain valid under much more general (risk averse) preferences. Similarly, while our information structure allows for the presence of both idiosyncratic and common informational noise components, it remains restrictive in the sense that both components are constrained to vanish at the same rate when the precision of private information goes to infinity (since the relation between the two is parameterised by the correlation coefficient $\rho$ ). We show formally in the online technical appendix that our results can be generalised to an information structure allowing each noise component to vanish at different rates. 


\section{APPENDIX}

A. Proof of Lemma 1 There are three aggregate shocks $(\theta, \tilde{\xi}, \varepsilon)$, hence three random variables that may affect $p$. Equation (1), implies that the effects of $\theta$ and $\alpha_{x}^{-1 / 2} \tilde{\xi}$ on private signals are indistinguishable. Hence we define $\tilde{\theta} \equiv \theta+\alpha_{x}^{-1 / 2} \tilde{\xi}$ and restrict our attention to equilibrium price functions $p(\tilde{\theta}, \varepsilon)$ that are linear in $(\tilde{\theta}, \varepsilon)$ (so that $p$ is normally distributed). A trader $i$ with risk aversion coefficient $\gamma_{i}$ and information set $G_{i}$ has a demand for assets $k_{i}\left(G_{i}\right)=\gamma_{i}^{-1} \mathbb{E}[\theta-$ $\left.p \mid G_{i}\right] / \mathbb{V}\left[\theta-p \mid G_{i}\right]$. We may thus write the demands by demand-schedule and market-order traders as $k_{I \backslash M}^{i}\left(x_{i}, p\right)=\gamma_{i}^{-1} f_{I \backslash M}\left(x_{i}, p\right)$ and $k_{M}^{i}\left(x_{i}\right)=\gamma_{i}^{-1} f_{M}\left(x_{i}\right)$, respectively, with

$$
f_{I \backslash M}\left(x_{i}, p\right)=\mathbb{E}\left[\theta-p \mid x_{i}, p\right] / \mathbb{V}\left[\theta \mid x_{i}, p\right], \quad f_{M}\left(x_{i}\right)=\mathbb{E}\left[\theta-p \mid x_{i}\right] / \mathbb{V}\left[\theta-p \mid x_{i}\right] .
$$

A.1. Price function. We conjecture that $f_{I \backslash M}, f_{M}$ have the form $f_{I \backslash M}\left(x_{i}, p\right)=a\left(x_{i}-\right.$ $\bar{\theta})+\zeta(p)$ and $f_{M}\left(x_{i}\right)=c\left(x_{i}-\bar{\theta}\right)$, where $a, c$ are normalised trading intensities (for a trader with $\left.\gamma_{i}=1\right)$ and $\zeta(\cdot)$ is a linear function. Using the convention that the average signal equals $\tilde{\theta}$ a.s., and recalling that $\gamma_{i}$ is independent from $\xi_{i}-\tilde{\xi}$, the order book is given by

$$
\begin{aligned}
L(p) & =\int_{I \backslash M} k_{I \backslash M}^{i}\left(x_{i}, p\right) \mathrm{d} i+\int_{M} k_{M}^{i}\left(x_{i}\right) \mathrm{d} i+\varepsilon=\int_{I \backslash M} \frac{a\left(x_{i}-\bar{\theta}\right)+\zeta(p)}{\gamma_{i}} \mathrm{~d} i+\int_{M} \frac{c\left(x_{i}-\bar{\theta}\right)}{\gamma_{i}} \mathrm{~d} i+\varepsilon \\
& =\left[a\left(\theta+\alpha_{x}^{-1 / 2} \tilde{\xi}-\bar{\theta}\right)\right] \int_{I \backslash M} \gamma_{i}^{-1} \mathrm{~d} i+c\left(\theta+\alpha_{x}^{-1 / 2} \tilde{\xi}-\bar{\theta}\right) \int_{M} \gamma_{i}^{-1} \mathrm{~d} i+\varepsilon+\zeta(p) \int_{I \backslash M} \gamma_{i}^{-1} \mathrm{~d} i \\
& =B\left(\theta+\alpha_{x}^{-1 / 2} \tilde{\xi}+B^{-1} \varepsilon\right)-B \bar{\theta}+\gamma_{I \backslash M}^{-1} \nu \zeta(p), \text { with } B=a \nu \gamma_{I \backslash M}^{-1}+c(1-\nu) \gamma_{M}^{-1} .
\end{aligned}
$$

The market making sector observes $L($.$) , a linear function of p$, and sets $p=\mathbb{E}[\theta \mid L()]=.\mathbb{E}[\theta \mid z]$, where $z=\theta+\alpha_{x}^{-1 / 2} \tilde{\xi}+B^{-1} \varepsilon$ summarises the information provided by the order book. Since $z$ provides a signal about $\theta$ with noise $\tilde{\varepsilon} \equiv \alpha_{x}^{-1 / 2} \tilde{\xi}+B^{-1} \varepsilon$ we have:

$$
p=\mathbb{E}[\theta \mid z]=\frac{\alpha_{\theta} \bar{\theta}+\alpha_{\tilde{\varepsilon}} z}{\alpha_{\theta}+\alpha_{\tilde{\varepsilon}}} \sim \mathcal{N}(\bar{\theta}, \mathbb{V}(p)), \text { where } \alpha_{\tilde{\varepsilon}}=\frac{1}{\mathbb{V}(\tilde{\varepsilon})}=\frac{B^{2} \alpha_{\varepsilon}}{1+\rho B^{2} \alpha_{\varepsilon} / \alpha_{x}}
$$

Rearranging the latter expression gives the price function (7) in Lemma 1.

A.2. Investment functions for $\rho \in(0,1)$. We now need to identify the parameters $a$ and $c$ in the demand functions, which requires computing the conditional moments of $\theta-p \mid G_{i}$, for $G_{i}=\left(x_{i}, p\right)$ (demand schedules) or $G_{i}=x_{i}$ (market orders). We start with the former and specifically focus on the moments of $\theta \mid x_{i}, p$ which is without loss of generality. To this purpose define $\boldsymbol{\Theta} \equiv\left[\begin{array}{llll}\theta & \tilde{\theta} & p & x_{i}\end{array}\right]^{\prime} \sim \mathcal{N}_{4}(\mathbb{E}(\boldsymbol{\Theta}), \mathbb{V}(\boldsymbol{\Theta}))$. From (1), (7) and the fact that $\tilde{\theta}=\theta+\alpha_{x}^{-1 / 2} \tilde{\xi}$, we have, for $\rho \in(0,1), \boldsymbol{\Theta}=\left[\begin{array}{llll}\bar{\theta} & \bar{\theta} & \bar{\theta} & \bar{\theta}\end{array}\right]^{\prime}+\mathbf{M S}$, with

$\mathbf{M}=\left[\begin{array}{cccc}\alpha_{\theta}^{-1 / 2} & 0 & 0 & 0 \\ \alpha_{\theta}^{-1 / 2} & \rho^{1 / 2} \alpha_{x}^{-1 / 2} & 0 & 0 \\ \lambda B \alpha_{\theta}^{-1 / 2} & \lambda B \rho^{1 / 2} \alpha_{x}^{-1 / 2} & \lambda \alpha_{\varepsilon}^{-1 / 2} & 0 \\ \alpha_{\theta}^{-1 / 2} & \rho^{1 / 2} \alpha_{x}^{-1 / 2} & 0 & (1-\rho)^{1 / 2} \alpha_{x}^{-1 / 2}\end{array}\right], \mathbf{S}=\left[\begin{array}{c}\alpha_{\theta}^{1 / 2}(\theta-\bar{\theta}) \\ \rho^{-1 / 2} \tilde{\xi} \\ \alpha_{\varepsilon}^{1 / 2} \varepsilon \\ (1-\rho)^{-1 / 2} \eta_{i}\end{array}\right] \sim \mathcal{N}_{4}(\mathbf{0}, \mathbf{I})$

Next, we compute $\mathbb{V}(\boldsymbol{\Theta})=\mathbf{M} \mathbf{M}^{\prime}$ and then partition $\mathbb{V}(\boldsymbol{\Theta})$ as $\mathbb{V}(\boldsymbol{\Theta})=\left[\Sigma_{k m}\right], k=1,2$, with all four $\Sigma_{k m}$ being $2 \times 2$ matrices. Then, from standard multivariate normal theory we know that 
$\left[\begin{array}{ll}\theta & \tilde{\theta}\end{array}\right]^{\prime} \mid p, x_{i}$ has distribution $\mathcal{N}_{2}\left(\left[\begin{array}{ll}\bar{\theta} & \bar{\theta}\end{array}\right]^{\prime}+\Sigma_{12} \Sigma_{22}^{-1}\left[\begin{array}{ll}p-\bar{\theta} & x_{i}-\bar{\theta}\end{array}\right]^{\prime}, \Sigma_{11}-\Sigma_{12} \Sigma_{22}^{-1} \Sigma_{21}\right)$, from which we infer the following conditional moments:

$$
\begin{aligned}
& \mathbb{E}\left[\theta \mid p, x_{i}\right]=\bar{\theta}+\frac{\alpha_{x}\left(x_{i}-\bar{\theta}\right)+(1-\rho) B^{2} \alpha_{\varepsilon}(\lambda B)^{-1}(p-\bar{\theta})}{(1-\rho)\left(1+\rho \alpha_{\theta} / \alpha_{x}\right) B^{2} \alpha_{\varepsilon}+\alpha_{x}+\alpha_{\theta}} \\
& \mathbb{V}\left[\theta \mid p, x_{i}\right]=\frac{1+(1-\rho) \rho B^{2} \alpha_{\varepsilon} / \alpha_{x}}{(1-\rho)\left(1+\rho \alpha_{\theta} / \alpha_{x}\right) B^{2} \alpha_{\varepsilon}+\alpha_{x}+\alpha_{\theta}}
\end{aligned}
$$

Substituting these values into $k_{I \backslash M}^{i}\left(x_{i}, p\right)=\gamma_{i}^{-1} \mathbb{E}\left[\theta-p \mid x_{i}, p\right] / \mathbb{V}\left[\theta \mid x_{i}, p\right]$ and rearranging gives the corresponding asset demand in Lemma 1.

We now turn to the computation of $\theta-p \mid x_{i}$. We define, still for $\rho \in(0,1), \boldsymbol{\Omega} \equiv\left[\begin{array}{llll}\theta & \tilde{\theta} & \theta-p & x_{i}\end{array}\right]^{\prime}$ $\sim \mathcal{N}_{4}(\mathbb{E}(\boldsymbol{\Omega}), \mathbb{V}(\boldsymbol{\Omega}))$ and note that $\boldsymbol{\Omega}=\left[\begin{array}{cccc}\bar{\theta} & \bar{\theta} & 0 & \bar{\theta}\end{array}\right]^{\prime}+\mathbf{N T}$, where

$$
\mathbf{N}=\left[\begin{array}{cccc}
\alpha_{\theta}^{-1 / 2} & 0 & 0 & 0 \\
\alpha_{\theta}^{-1 / 2} & \rho^{1 / 2} \alpha_{x}^{-1 / 2} & 0 & 0 \\
(1-\lambda B) \alpha_{\theta}^{-1 / 2} & -\lambda B \rho^{1 / 2} \alpha_{x}^{-1 / 2} & -\lambda \alpha_{\varepsilon}^{-1 / 2} & 0 \\
\alpha_{\theta}^{-1 / 2} & \rho^{1 / 2} \alpha_{x}^{-1 / 2} & 0 & (1-\rho)^{1 / 2} \alpha_{x}^{-1 / 2}
\end{array}\right], \mathbf{T}=\left[\begin{array}{c}
\alpha_{\theta}^{1 / 2}(\theta-\bar{\theta}) \\
\rho^{-1 / 2} \tilde{\xi} \\
\alpha_{\varepsilon}^{1 / 2} \varepsilon \\
(1-\rho)^{-1 / 2} \eta_{i}
\end{array}\right]
$$

so that $\mathbf{T} \sim \mathcal{N}_{4}(\mathbf{0}, \mathbf{I})$. We partition $\mathbb{V}(\boldsymbol{\Omega})=\mathbf{N N}^{\prime}$ as follows: $\mathbb{V}(\boldsymbol{\Omega})=\left[\bar{\Sigma}_{k m}\right], k, m=1$, 2 , where $\bar{\Sigma}_{11}$ is $3 \times 3, \bar{\Sigma}_{12}$ is $3 \times 1, \bar{\Sigma}_{21}$ is $1 \times 3$ and $\bar{\Sigma}_{22}$ is $1 \times 1$. It follows that [ $\left.\theta \quad \tilde{\theta} \theta-p\right]^{\prime} \mid x_{i}$ has distribution $\mathcal{N}_{3}\left(\left[\begin{array}{lll}\bar{\theta} & \bar{\theta} & 0\end{array}\right]^{\prime}+\bar{\Sigma}_{12} \bar{\Sigma}_{22}^{-1}\left(x_{i}-\bar{\theta}\right), \bar{\Sigma}_{11}-\bar{\Sigma}_{12} \bar{\Sigma}_{22}^{-1} \bar{\Sigma}_{21}\right)$. After some calculations, we infer from this joint distribution that:

$$
\begin{aligned}
& \mathbb{E}\left[\theta-p \mid x_{i}\right]=\frac{\left((1-\lambda B) \alpha_{\theta}^{-1}-\lambda B \rho \alpha_{x}^{-1}\right)\left(x_{i}-\bar{\theta}\right)}{\alpha_{\theta}^{-1}+\alpha_{x}^{-1}} \\
& \mathbb{V}\left[\theta-p \mid x_{i}\right]=(\lambda B)^{2}\left(\frac{\left(\rho+\rho \alpha_{\theta} / \alpha_{x}+B^{-2} \alpha_{\theta} / \alpha_{\varepsilon}\right)^{2}}{\alpha_{x}+\alpha_{\theta}}+\frac{(1-\rho) \rho}{\alpha_{x}}+\frac{1}{B^{2} \alpha_{\varepsilon}}\right)
\end{aligned}
$$

Substituting (A3)-(A4) into $k_{M}^{i}\left(x_{i}\right)=\gamma_{i}^{-1} \mathbb{E}\left[\theta-p \mid x_{i}\right] / \mathbb{V}\left[\theta-p \mid x_{i}\right]$ and rearranging gives the asset demand of market-order traders in Lemma 1.

A.3. Investment functions for $\rho \in\{0,1\}$. The expressions for $f_{I \backslash M}\left(x_{i}, p\right), f_{M}\left(x_{i}\right)$, which have been derived for $\rho \in(0,1)$, can be extended by continuity to $\rho \in\{0,1\}$. For example, for $\rho=0$ we have $z=\theta+B^{-1} \varepsilon$, and computing the joint distribution of $\left(p, x_{i}, \theta\right)$ gives the same conditional moments as those in (A1)-(A4) when setting $\rho=0$. Similarly, when $\rho=1$ all informed traders receive the same signal $x=\theta+\alpha_{x}^{-1 / 2} \tilde{\xi}, \tilde{\xi} \sim \mathcal{N}(0,1)$, hence observing $p$ does not provide any more information about $\theta$ than observing $x$. It follows that:

$$
\mathbb{E}[\theta \mid p, x]=\mathbb{E}[\theta \mid x]=\left(\alpha_{\theta} \bar{\theta}+\alpha_{x} x\right) /\left(\alpha_{\theta}+\alpha_{x}\right), \mathbb{V}[\theta \mid p, x]=\mathbb{V}[\theta \mid x]=\left(\alpha_{x}+\alpha_{\theta}\right)^{-1},
$$

which is recovered by setting $\rho=1$ in (A1)-(A4). Hence the expressions for $k_{I \backslash M}\left(x_{i}, p ; \gamma_{i}\right)$, $k_{M}\left(x_{i} ; \gamma_{i}\right)$ in Lemma 1 are valid for $\rho \in(0,1) \cup\{0,1\}=[0,1]$. 
A.4. Uniqueness of linear equilibrium. We finally show that $B$ is unique, positive and finite. To do this, define the function $f: \mathbb{R} \rightarrow \mathbb{R}$ as

$f(B)=\frac{\nu \alpha_{x}}{\gamma_{I \backslash M}\left(1+\frac{(1-\rho) \rho B^{2} \alpha_{\varepsilon}}{\alpha_{x}}\right)}+\frac{1-\nu}{\gamma_{M}\left(\left(\alpha_{x}^{-1}+\alpha_{\theta}^{-1}\right)\left(1+B^{2} \frac{\rho \alpha_{\varepsilon}}{\alpha_{x}}\right)-\left(\alpha_{\theta}+B^{2} \alpha_{\varepsilon}\left(1+\frac{\rho \alpha_{\theta}}{\alpha_{x}}\right)\right)^{-1}\right)}-B$,

so that a root of $f(B)$ solves (9). $f$ is continuous and strictly decreasing over $[0,+\infty)$ and such that $f(0)=\frac{\nu \alpha_{x}}{\gamma_{I \backslash M}}+(1-\nu) \frac{\alpha_{x}}{\gamma_{M}}>0$ and $f(+\infty)=-\infty$. Hence $f$ is a bijection with a unique root $B_{0}>0$ over $[0,+\infty)$. Since $f$ is strictly positive on $\mathbb{R}^{-}, B_{0}$ is the unique root of $f$ in $\mathbb{R}$.

B. Proof of Proposition 1 From (7), $p$ is observationally equivalent to $z=\theta+\alpha_{x}^{-1 / 2} \tilde{\xi}+B^{-1} \varepsilon$, so both provide the same information about $\theta$. It follows that the precision of the price signal is:

$$
\alpha_{p}=\alpha_{z}=1 / \mathbb{V}\left(\alpha_{x}^{-1 / 2} \tilde{\xi}+B^{-1} \varepsilon\right)=1 /\left(\rho \alpha_{x}^{-1}+B^{-2} \alpha_{\varepsilon}^{-1}\right)
$$

B.1. Full market-order case. We know from Lemma 1 that $B>0$ uniquely solves (9). Now define the function $g: \mathbb{R}_{+}^{*} \times \mathbb{R}_{+}^{*} \rightarrow \mathbb{R}$ as follows:

$$
g\left(B, \alpha_{x}\right)=\gamma_{I}^{-1}\left[\left(\alpha_{x}^{-1}+\alpha_{\theta}^{-1}\right)\left(1+B^{2} \rho \alpha_{\varepsilon} / \alpha_{x}\right)-\left(\alpha_{\theta}+B^{2} \alpha_{\varepsilon}\left(1+\rho \alpha_{\theta} / \alpha_{x}\right)\right)^{-1}\right]^{-1}-B
$$

When $\nu=0, B$ is the unique solution to $g\left(B, \alpha_{x}\right)=0$. Since $g$ is continuously differentiable, increasing in $a_{x}$ and decreasing in $B$ on $\mathbb{R}_{+}^{*} \times \mathbb{R}_{+}^{*}$, the implicit function $B\left(\alpha_{x}\right)$ defined by $g\left(B, \alpha_{x}\right)=$ 0 is continuously differentiable and increasing over $\mathbb{R}_{+}^{*}$. Moreover, we have:

$$
\begin{aligned}
\left(\alpha_{x}^{-1}+\alpha_{\theta}^{-1}\right) & \left(1+B^{2} \rho \alpha_{\varepsilon} / \alpha_{x}\right)-\left(\alpha_{\theta}+B^{2} \alpha_{\varepsilon}\left(1+\rho \alpha_{\theta} / \alpha_{x}\right)\right)^{-1} \\
& \geq \alpha_{\theta}^{-1}-\left(B^{2} \alpha_{\varepsilon}\left(1+\rho \alpha_{\theta} / \alpha_{x}\right)+\alpha_{\theta}\right)^{-1} \geq \alpha_{\theta}^{-1}-\left(B^{2} \alpha_{\varepsilon}+\alpha_{\theta}\right)^{-1} \\
\text { so that } \quad & B=\gamma_{I}^{-1}\left[\left(\alpha_{x}^{-1}+\alpha_{\theta}^{-1}\right)\left(1+B^{2} \rho \alpha_{\varepsilon} / \alpha_{x}\right)-\left(\alpha_{\theta}+B^{2} \alpha_{\varepsilon}\left(1+\rho \alpha_{\theta} / \alpha_{x}\right)\right)^{-1}\right]^{-1} \\
\leq & \gamma_{I}^{-1}\left[\alpha_{\theta}^{-1}-\left(B^{2} \alpha_{\varepsilon}+\alpha_{\theta}\right)^{-1}\right]^{-1}=\gamma_{I}^{-1} \alpha_{\theta}\left(B^{2} \alpha_{\varepsilon}+\alpha_{\theta}\right) \leq \gamma_{I}^{-1} \alpha_{\theta}\left(1+\alpha_{\theta} B^{-2} \alpha_{\varepsilon}^{-1}\right)
\end{aligned}
$$

The function $h_{+}: B \rightarrow B-\gamma_{I}^{-1} \alpha_{\theta}\left(1+\alpha_{\theta} / B^{2} \alpha_{\varepsilon}\right)$ is continuous and strictly increasing over $\mathbb{R}_{+}^{*}$, and such that $h_{+}(0)=-\infty$ and $h_{+}(+\infty)=+\infty$. It is thus bijective and we denote its inverse by $h_{+}^{-1}$. Then $\left.\left.B \in h_{+}^{-1}(]-\infty ; 0\right]\right)$ is bounded above by $h_{+}^{-1}(0)$, which is positive and independent of $\alpha_{x}$. Hence, $B_{0} \equiv \lim _{\alpha_{x} \rightarrow \infty} B\left(\alpha_{x}\right)$ is defined and, by continuity, is the unique solution to $\gamma_{I} B=\left(\alpha_{\theta}^{-1}-\left(\alpha_{\theta}+\alpha_{\varepsilon} B^{2}\right)^{-1}\right)^{-1}$. From (7) we then infer that $\lim _{\alpha_{x} \rightarrow \infty} \alpha_{p}=B_{0}^{2} \alpha_{\varepsilon}$.

B.2. Other cases. When $\nu \in(0,1], B\left(\alpha_{x}\right)$ is implicitly defined as the unique solution to (9). When $\rho \in\{0,1\}$, we have

$$
\frac{B\left(\alpha_{x}\right)}{\frac{\nu \alpha_{x}}{\gamma_{I \backslash M}}}=1+\frac{(1-\nu) / \gamma_{M}}{\left(\alpha_{x}^{-1}+\alpha_{\theta}^{-1}\right)\left(1+B^{2} \rho \alpha_{\varepsilon} / \alpha_{x}\right)-\left(B\left(\alpha_{x}\right)^{2} \alpha_{\varepsilon}\left(1+\rho \alpha_{\theta} / \alpha_{x}\right)+\alpha_{\theta}\right)^{-1}} \frac{1}{\frac{\nu \alpha_{x}}{\gamma_{I \backslash M}}}
$$

so that $B\left(\alpha_{x}\right) \geq \nu \alpha_{x} / \gamma_{I \backslash M}$. Hence, whenever $\rho \in\{0,1\}$ and $\nu>0$ we have $\lim _{\alpha_{x} \rightarrow+\infty} B\left(\alpha_{x}\right)=$ $+\infty$ and $\lim _{\alpha_{x} \rightarrow+\infty}\left[B\left(\alpha_{x}\right)\right]^{2} / \alpha_{x}=+\infty$. 
It follows that $\lim _{\alpha_{x} \rightarrow+\infty} B\left(\alpha_{x}\right) /\left(\frac{\nu \alpha_{x}}{\gamma_{I \backslash M}}\right)$ is equal to

$$
1+\lim _{\alpha_{x} \rightarrow+\infty} \frac{(1-\nu) / \gamma_{M}}{\left(\alpha_{x}^{-1}+\alpha_{\theta}^{-1}\right)\left(1+B\left(\alpha_{x}\right)^{2} \rho \alpha_{\varepsilon} / \alpha_{x}\right)-\left(B\left(\alpha_{x}\right)^{2} \alpha_{\varepsilon}\left(1+\rho \alpha_{\theta} / \alpha_{x}\right)+\alpha_{\theta}\right)^{-1}} \frac{1}{\frac{\nu \alpha_{x}}{\gamma_{I \backslash M}}}=1,
$$

so that $B\left(\alpha_{x}\right) \underset{\alpha_{x} \rightarrow+\infty}{\sim} \nu \alpha_{x} / \gamma_{I \backslash M}$. Now, recall from (7) that $\alpha_{p}=\left(\rho / \alpha_{x}+B^{-2} / \alpha_{\varepsilon}\right)^{-1}$. Hence, for $\rho=0$ we have $\lim _{\alpha_{x} \rightarrow+\infty} \alpha_{p}=\lim _{\alpha_{x} \rightarrow+\infty}\left[B\left(\alpha_{x}\right)\right]^{2} \alpha_{\varepsilon}=\left(\nu \alpha_{x} / \gamma_{I \backslash M}\right)^{2} \alpha_{\varepsilon}$, that is $\alpha_{p} \underset{\alpha_{x} \rightarrow+\infty}{\sim}$ $\left(\nu / \gamma_{I \backslash M}\right)^{2} \alpha_{\varepsilon} \alpha_{x}^{2}$. However, for $\rho=1$ we have $\alpha_{p} \underset{\alpha_{x} \rightarrow+\infty}{\sim} \alpha_{x}$. Indeed, in this case we have $\alpha_{p}^{-1}=$ $\alpha_{x}^{-1}+B^{-2} \alpha_{\varepsilon}^{-1}$, and we know that $\lim _{\alpha_{x} \rightarrow+\infty} B^{-2} \alpha_{\varepsilon}^{-1} / \alpha_{x}^{-1}=\alpha_{\varepsilon}^{-1} \lim _{\alpha_{x} \rightarrow+\infty} \alpha_{x} / B^{2}=0$, from which it follows that $\alpha_{p}^{-1} \underset{\alpha_{x} \rightarrow+\infty}{\sim} \alpha_{x}^{-1}$.

Let us now turn to the case where $\rho \in(0,1)$. In that situation (9) implies that

$$
B\left(\alpha_{x}\right) \geq \frac{\nu \alpha_{x}}{\gamma_{I \backslash M}} \frac{1}{1+(1-\rho) \rho B\left(\alpha_{x}\right)^{2} \alpha_{\varepsilon} / \alpha_{x}} \Leftrightarrow \frac{B\left(\alpha_{x}\right)}{\alpha_{x}^{1 / 2}}\left(1+(1-\rho) \rho B\left(\alpha_{x}\right)^{2} \alpha_{\varepsilon} / \alpha_{x}\right) \geq \frac{\nu \alpha_{x}^{1 / 2}}{\gamma_{I \backslash M}},
$$

so that $\lim _{\alpha_{x} \rightarrow \infty} B\left(\alpha_{x}\right) / \alpha_{x}^{1 / 2}=\lim _{\alpha_{x} \rightarrow \infty} B\left(\alpha_{x}\right)^{2} / \alpha_{x}=+\infty$. Moreover, again from (9) we have

$$
\frac{B\left(\alpha_{x}\right)^{3}}{\alpha_{x}^{2}}=\frac{\nu / \gamma_{I \backslash M}}{\alpha_{x} / B\left(\alpha_{x}\right)^{2}+(1-\rho) \rho \alpha_{\varepsilon}}+\frac{\left[(1-\nu) / \gamma_{M}\right] \times B\left(\alpha_{x}\right)^{2} / \alpha_{x}^{2}}{\left(\frac{1}{\alpha_{x}}+\frac{1}{\alpha_{\theta}}\right)\left(1+\frac{B\left(\alpha_{x}\right)^{2} \rho \alpha_{\varepsilon}}{\alpha_{x}}\right)-\left(B\left(\alpha_{x}\right)^{2} \alpha_{\varepsilon}\left(1+\frac{\rho \alpha_{\theta}}{\alpha_{x}}\right)+\alpha_{\theta}\right)^{-1}}
$$

Now, since

$$
\frac{\left[(1-\nu) / \gamma_{M}\right] \times B\left(\alpha_{x}\right)^{2} / \alpha_{x}^{2}}{\left(\frac{1}{\alpha_{x}}+\frac{1}{\alpha_{\theta}}\right)\left(1+\frac{B\left(\alpha_{x}\right)^{2} \rho \alpha_{\varepsilon}}{\alpha_{x}}\right)-\left(B\left(\alpha_{x}\right)^{2} \alpha_{\varepsilon}\left(1+\frac{\rho \alpha_{\theta}}{\alpha_{x}}\right)+\alpha_{\theta}\right)^{-1}} \underset{\alpha_{x} \rightarrow \infty}{\rightarrow} 0 \text { and } \frac{\alpha_{x}}{B\left(\alpha_{x}\right)^{2}} \underset{\alpha_{x} \rightarrow \infty}{\rightarrow} 0
$$

we get

$$
\frac{B^{3}\left(\alpha_{x}\right)}{\alpha_{x}^{2}} \underset{\alpha_{x} \rightarrow \infty}{\rightarrow} \frac{\nu / \gamma_{I \backslash M}}{(1-\rho) \rho \alpha_{\varepsilon}} \Rightarrow B\left(\alpha_{x}\right) \underset{\alpha_{x} \rightarrow \infty}{\sim}\left(\frac{\nu \alpha_{x}^{2} / \gamma_{I \backslash M}}{(1-\rho) \rho \alpha_{\varepsilon}}\right)^{1 / 3} .
$$

Recall that $\alpha_{p}^{-1}=\rho \alpha_{x}^{-1}+B^{-2} \alpha_{\varepsilon}^{-1}$, and we have shown that $\lim _{\alpha_{x} \rightarrow \infty} B\left(\alpha_{x}\right)^{2} / \alpha_{x}=+\infty$. Hence, $\lim _{\alpha_{x} \rightarrow \infty} \alpha_{x} / B\left(\alpha_{x}\right)^{2}=0$, so that $\alpha_{p}^{-1} \underset{\alpha_{x} \rightarrow \infty}{\sim} \rho \alpha_{x}^{-1}$.

C. Proof of Lemma 2 Let us first state the version of the law of total variance that is relevant in our context:

$$
\begin{gathered}
\mathbb{V}\left[\mathbb{E}\left[\theta-p \mid x_{i}, p\right] \mid x_{i}\right]=\mathbb{V}\left[\theta-p \mid x_{i}\right]-\mathbb{E}\left[\mathbb{V}\left[\theta-p \mid x_{i}, p\right] \mid x_{i}\right]=\mathbb{V}\left[\theta-p \mid x_{i}\right]-\mathbb{V}\left[\theta-p \mid x_{i}, p\right] \\
\text { and } \mathbb{V}\left[\mathbb{E}\left[\theta-p \mid x_{i}\right]\right]=\mathbb{V}[\theta-p]-\mathbb{E}\left[\mathbb{V}\left[\theta-p \mid x_{i}\right]\right]=\mathbb{V}[\theta-p]-\mathbb{V}\left[\theta-p \mid x_{i}\right]
\end{gathered}
$$

C.1. Timing 1: Order type chosen after the signal is observed Under timing 1 traders observe $x_{i}$, know that the price will be a linear function of normally distributed variables, and then compare $W_{M}\left(x_{i} ; \gamma_{i}\right)$ and $\mathbb{E}\left[W_{I \backslash M}\left(x_{i}, p ; \gamma_{i}\right) \mid x_{i}\right]$. We first write $W_{M}(\cdot)$ as

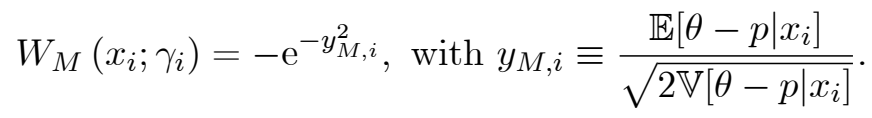


Similarly, $W_{I \backslash M}\left(x_{i}, p ; \gamma_{i}\right)=-\mathrm{e}^{-y_{I \backslash M, i}^{2}+c \gamma_{i}}$, with $y_{I \backslash M, i} \equiv \mathbb{E}\left[\theta-p \mid x_{i}, p\right] / \sqrt{2 \mathbb{V}\left[\theta-p \mid x_{i}, p\right]}$ and

$$
y_{I \backslash M, i} \mid x_{i} \sim \mathcal{N}\left(\frac{\mathbb{E}\left[\theta-p \mid x_{i}\right]}{\sqrt{2 \mathbb{V}\left[\theta-p \mid x_{i}, p\right]}}, \frac{\mathbb{V}\left[\mathbb{E}\left[\theta-p \mid x_{i}, p\right] \mid x_{i}\right]}{2 \mathbb{V}\left[\theta-p \mid x_{i}, p\right]}\right) .
$$

Using the fact that $y_{I \backslash M, i}^{2} \mid x_{i}$ has a noncentral chi-square distribution (see, e.g., Grossman and Stiglitz, 1980), the moment-generating function yields:

$$
\begin{aligned}
& \mathbb{E}\left[W_{I \backslash M}\left(x_{i}, p ; \gamma_{i}\right) \mid x_{i}\right] \\
& =-\mathbb{E}\left[\mathrm{e}^{-y_{I \backslash M, i}^{2}+c \gamma_{i}} \mid x_{i}\right]=-\frac{\mathrm{e}^{c \gamma_{i}}}{\sqrt{1+2 \mathbb{V}\left[y_{I \backslash M, i} \mid x_{i}\right]}} \exp \left(-\frac{\left(\mathbb{E}\left[y_{I \backslash M, i} \mid x_{i}\right]\right)^{2}}{1+2 \mathbb{V}\left[y_{I \backslash M, i} \mid x_{i}\right]}\right) \\
& =-\mathrm{e}^{c \gamma_{i}}\left(\frac{\mathbb{V}\left[\theta-p \mid x_{i}, p\right]}{\mathbb{V}\left[\theta-p \mid x_{i}, p\right]+\mathbb{V}\left[\mathbb{E}\left[\theta-p \mid x_{i}, p\right] \mid x_{i}\right]}\right)^{1 / 2} \exp \left(-\frac{1}{2} \frac{\left(\mathbb{E}\left[\theta-p \mid x_{i}\right]\right)^{2}}{\mathbb{V}\left[\theta-p \mid x_{i}, p\right]+\mathbb{V}\left[\mathbb{E}\left[\theta-p \mid x_{i}, p\right] \mid x_{i}\right]}\right) \\
& =-\mathrm{e}^{c \gamma_{i}} \sqrt{\frac{\mathbb{V}\left[\theta-p \mid x_{i}, p\right]}{\mathbb{V}\left[\theta-p \mid x_{i}\right]}} \exp \left(-\frac{1}{2} \frac{\left(\mathbb{E}\left[\theta-p \mid x_{i}\right]\right)^{2}}{\mathbb{V}\left[\theta-p \mid x_{i}\right]}\right)
\end{aligned}
$$

where we have used (C1) to go from the second to the third line. Comparing (C3) and (C4) gives the first ratio in Lemma 2.

C2. Timing 2: Order type chosen before the signal is observed Under timing 2, traders choose their order type before knowing $x_{i}$. We recover the relevant expected utility levels by integrating those under timing 1 over $x_{i}$. First, noting that $y_{M, i}^{2}$ has a noncentral chi-square distribution and making use of (C2), we obtain:

$$
\mathbb{E}\left[W_{M}\left(x_{i} ; \gamma_{i}\right)\right]=\mathbb{E}\left[-\mathrm{e}^{\left.-y_{M, i}^{2}\right]}=-\left(1+\frac{\mathbb{V}\left[\mathbb{E}\left[\theta-p \mid x_{i}\right]\right]}{\mathbb{V}\left[\theta-p \mid x_{i}\right]}\right)^{-1 / 2}=-\left(\frac{\mathbb{V}[\theta-p]}{\mathbb{V}\left[\theta-p \mid x_{i}\right]}\right)^{-1 / 2} .\right.
$$

Now, using the law of iterated expectations and integrating (C4) over $x_{i}$ we get:

$$
\begin{aligned}
\mathbb{E}\left[W_{I \backslash M}\left(x_{i}, p ; \gamma_{i}\right)\right] & =\mathbb{E}\left[\mathbb{E}\left[W_{I \backslash M}\left(x_{i}, p ; \gamma_{i}\right) \mid x_{i}\right]\right] \\
& =-\mathrm{e}^{c \gamma_{i}}\left(\frac{\mathbb{V}\left[\theta-p \mid x_{i}, p\right]}{\mathbb{V}\left[\theta-p \mid x_{i}\right]}\right)^{1 / 2} \mathbb{E}\left[\exp \left(-\frac{1}{2} \frac{\left(\mathbb{E}\left[\theta-p \mid x_{i}\right]\right)^{2}}{\mathbb{V}\left[\theta-p \mid x_{i}\right]}\right)\right]
\end{aligned}
$$

But again, $\left(\mathbb{E}\left[\theta-p \mid x_{i}\right]\right)^{2}$ also has a noncentral chi-square distribution and we have:

$$
\mathbb{E}\left[\exp \left(\frac{-\left(\mathbb{E}\left[\theta-p \mid x_{i}\right]\right)^{2}}{2 \mathbb{V}\left[\theta-p \mid x_{i}\right]}\right)\right]=\left(1+\frac{\mathbb{V}\left[\mathbb{E}\left[\theta-p \mid x_{i}\right]\right]}{\mathbb{V}\left[\theta-p \mid x_{i}\right]}\right)^{-1 / 2} .
$$

Substituting (C7) into (C6), making use of $(\mathrm{C} 2)$ and rearranging, we get:

$$
\mathbb{E}\left[W_{I \backslash M}\left(x_{i}, p ; \gamma_{i}\right)\right]=-\mathrm{e}^{c \gamma_{i}}\left(\frac{\mathbb{V}[\theta-p]}{\mathbb{V}\left[\theta-p \mid x_{i}, p\right]}\right)^{-1 / 2} .
$$

Comparing (C5) and (C8) gives the second ratio in Lemma 2.

D. Proof of Proposition 2 See the separate online technical appendix. 


\section{REFERENCES}

[1] Angeletos, G.-M. and Werning, I. (2006), Crises and prices: information aggregation, multiplicity, and volatility, American Economic Review, 96(5), 1720-1736.

[2] Bai Y., Chang E., and Wang J. (2006), Asset prices under short-sale constraints. MIT WP.

[3] Barlevy, G. and Veronesi, P. (2000), Information acquisition in financial markets, Review of Economic Studies, 67, pp.79-90.

[4] Brown, D.P. and Zhang, Z.M. (1997), Market orders and market efficiency, Journal of Finance, 52(1), 277-308.

[5] Diamond, D.W. and Verrecchia, R.E. (1981), Information aggregation in a noisy rational expectations economy, Journal of Financial Economics, 9, pp. 231-235.

[6] Diamond, D.W. and Verrecchia, R.E. (1987), Constraints on short-selling and asset price adjustment to private information, Journal of Financial Economics, 18, 277-311

[7] Grossman, S.J. and Stiglitz, J. (1980), On the impossibility of informationally efficient markets, American Economic Review, 70(3), 393-408.

[8] Grossman, S.J. and Stiglitz, J. (1976), Information and competitive price system, American Economic Review, 66(2), 246-53.

[9] Kyle, A.S. (1989), Informed speculation under imperfect competition, Review of Economic Studies, 56(3), 317-355.

[10] Medrano, L.A. (1996), Market versus limit orders in an imperfectly competitive security market, UPF Working paper 165, february.

[11] Miller E. (1977), Risk, uncertainty and divergence of opinion. Journal of Finance, 32, 11511168.

[12] Peress, J. (2004), The tradeoff between risk sharing and information production in financial markets, Journal of Economic Theory, 145(1), 124-155.

[13] Verrecchia, R.E. (1982), Information acquisition in a noisy rationial expectation economy, Econometrica, 50(6), pp. 1415-1430.

[14] Vives, X. (2013), On the possibility of informationally efficient markets: A large-market rational expectations equilibrium model“, CEPR DP 6720.

[15] Vives, X. (2008), Information and Learning in Markets: The Impact of Market Microstructure, Princeton University Press.

[16] Vives, X. (1995), The speed of information revelation in a financial market mechanism, Journal of Economic Theory, 67(1), 178-204.

[17] Wald, J.K. and Horrigan, H.T. (2005), Optimal limit order choice, Journal of Business, 78(2), 597-619. 